\title{
Mapping Knowledge Management and Organizational Learning in Support of Organizational Memory
}

\author{
Zahir Irani ${ }^{1}$, Amir M. Sharif and Peter E.D. Love ${ }^{2}$ \\ ${ }^{1}$ Brunel Business School \\ Brunel University \\ Uxbridge, UB8 3PH, UK \\ ${ }^{2}$ Curtin University of Technology \\ GPO Box 1987 \\ Perth, WA 6845, Australia
}

\begin{abstract}
The normative literature within the field of Knowledge Management has concentrated on techniques and methodologies for allowing knowledge to be codified and made available to individuals and groups within organizations. The literature on Organizational Learning however, has tended to focus on aspects of knowledge that are pertinent at the macro-organizational level (i.e. the overall business). The authors attempt in this paper to address a relative void in the literature, aiming to demonstrate the inter-locking factors within an enterprise information system that relate knowledge management and organizational learning, via a model that highlights key factors within such an inter-relationship. This is achieved by extrapolating data from a manufacturing organization using a case study, with these data then modeled using a cognitive mapping technique (Fuzzy Cognitive Mapping, FCM). The empirical enquiry explores an interpretivist view of knowledge, within an Information Systems Evaluation (ISE) process, through the associated classification of structural, interpretive and evaluative knowledge. This is achieved by visualizng inter-relationships within the ISE decision-making approach in the case organization. A number of decision paths within the cognitive map are then identified such that a greater understanding of ISE can be sought. The authors therefore present a model that defines a relationship between Knowledge Management $(\mathrm{KM})$ and Organisational Learning (OL), and highlights factors that can lead a firm to develop itself towards a learning organization.
\end{abstract}

Keywords: Knowledge Management, Organizational Learning, Fuzzy Cognitive Mapping, Case Study

\footnotetext{
${ }^{1}$ Corresponding Author: Zahir.Irani@Brunel.ac.uk
} 


\section{Introduction}

Dynamic theory of organizational knowledge introduced by Nonaka (1994), explains that organizational knowledge is created through a continuous dialogue between tacit and explicit knowledge. Such interaction takes place through four touch-points, namely, socialization, combination, internalization and externalization. Such interaction is transcendental, and as Sorensen and Kakihara (2002) explain, simply encoding data in itself, does not provide a context for using information and the harnessing of knowledge, effectively. There is a pressing need to understand the manner by which one uses and can learn from such information (i.e. the sublimation of information into useful data, or knowledge) codified and made accessible via an Information System (IS). This need is often motivated by the desire to exploit data and its opportunity to craft information and thus knowledge. It is postulated that whilst Organizational Learning (OL) might be the ideal that organizations want to accomplish, Knowledge Management (KM) is the reality of what can be achieved. Indeed, King et al. (2002) note the results of a survey conducted by senior knowledge managers that reports the vast majority of practitioners focus their attention on the strategic management of learning, and harnessing of knowledge. In furtherance of such findings, there remains a need to not only define the contingent difference between knowledge management and organizational learning but also to provide an insight into those organizational factors that can support a business towards becoming a learning organization; where people at all levels within the business, individually and collectively, increasing their capacity to improve their personal and professional performance.

This paper derives its impetus and motivation through an established void in the literature that supports the need for an integrated model for KM and its relationship with $\mathrm{OL}$, which in doing so, highlights behavioral and process issues surrounding Information Systems Evaluation (ISE). The authors present the development of a model that highlights the factors for such an inter-relationship, which evolves from a case study research strategy that exploits qualitative information that is then modeled using FCM. While previous research showed that experience and intuition often served as the primary evaluation criteria (Kaplan, 1986; Lohse et al., 1995; Irani and Love, 2001), this empirical enquiry explores an interpretivist view of knowledge, within an IT/IS investment evaluation decision-making process. Through a classification of knowledge into three forms, structural, interpretive and evaluative, the given case attempts to elucidate the inherent, underlying explicit/tacit knowledge relationships that define key KM to OL factors.

The authors seek to establish such relationships through the application of a cognitive mapping technique; Fuzzy Cognitive Mapping (FCM), to visualize aspects of the organization's decisionmaking approach, which leads to the identification of contingent knowledge factors and dependencies. In doing so, examining the dynamics of knowledge and the role that knowledge plays within the maturity of a learning organization. The authors seek to analyze the nature of explicit and tacit knowledge inter-relationships that exist within the ISE process, as a result of the FCM and case data. Such lucid tacit knowledge flows will, in turn, give way to the exploration of those aspects that may give rise to organizational learning within the context of a manufacturing firm. The conclusions are not seeking to offer generality but to allow others to draw parallels in constants and processes and thus, be supportive of decision-making processes. 


\section{Knowledge Management and Organizational Learning}

Information Systems Evaluation (ISE) seeks to provide an understanding of decision-making tasks through a mapping of core factors to the investment justification process. Hence, there is a need to not only understand basic business principles but also a need to understand the specific nuances of a particular business case. Hence, the underlying theme of ISE is the influence of organizational culture and learning, on the decision-making of individuals. This has been a topic of much discussion, by authors such as Skerlevaj et al. (2007), who note that an organizational learning culture is impacted by the transformation of information across both internal and external company boundaries and environments that often results from investments. Kess and Haapasalo (2002) also note the importance of including and imbibing knowledge and the review of organizational information within production-based company, if nothing else giving the organization "something to learn". Saunders and Miranda (1998) expressed the criticality of acquiring and using information in the decision-making process, yet the form and type of knowledge required to make investment decisions has not generally been focused upon in the normative literature. Rather, there has been a tendency to pay attention to the methods and techniques employed via traditional cost accounting and financial methods (Ballantine and Stray, 1999; Irani et al., 1997). This is somewhat surprising given that the knowledge and experiential learning that is required within this decisionmaking process, is crucial to the outcome. It is here where this paper seeks to make a significant contribution to the extant literature, through providing a deeper understanding of the relationship that exists between KM and OL, through the use of an FCM approach (when contextualized within an ISE process).

Advances in implementing information systems within the manufacturing sector, and the evolution and progression of cheaply available computing power, has meant that many manufactured goods and / or services, are now equally dependent upon the input of information and knowledge resources. In fact, information itself is becoming the product being sold, as explained by Järvenpää and Immonen (1998). Hence, over the years, it has also become increasingly important to understand the manner by which such resources are applied to, and used within the organizational context. The growth of the field of knowledge management has therefore seen the development of processes and tools that address the codification, collaboration, dissemination and representation of knowledge.

Nonaka and Takeuchi (1995) famously built upon the ideas of Polanyi, differentiating between so-called expressible, explicit knowledge; and inarticulable, tacit knowledge (knowledge which we find difficult to articulate). Whilst these approaches have been somewhat successful as compared to the purely structural and interpretive forms of knowledge, there remains a need to understand the context of how knowledge is used, specifically for human decision-making tasks. More importantly, the creation of internalized, organizational knowledge has been understood to be because of individual and collective experiences within the firm (Schumpeter, 1934). Thus, the concept of an organizational or "corporate memory" (Handy, 1990) has also become a very powerful and ubiquitous concept, and is seen by many as being an aspect of knowledge itself, being termed Organizational Learning (OL). As King (2000) and King and Ko (2001) note, there is much confusion over knowledge management and organizational learning terms, which are often used 
interchangeably however, Levine (2001) and Sunassee et al. (1965) stressed the contingent differences between organizational learning; and a learning organization. For an organization to become one that is a learning entity requires it to overcome barriers of individual / team learning; be able to arrive at a common understanding of company purpose and known organizational problems; and exhibit a certain level of error tolerance (i.e. incorrect learning or learning from bad / critical experiences). As Sunassee et al. note, learning within these contexts can only occur at an individual level (where change is subsumed as part of work practices) or at an organizational level (where a technology management change strategy drives all other managerial initiatives). This has also been highlighted by Irani et al. (2005), where the authors have attempted to elucidate this via specific mapping of knowledge in a process sense - and also in terms of "techno change" projects, by Markus (2004). Whilst, in essence, the organizational context of learning is ultimately driven by the dynamics of knowledge transfer and discourse (in a social sense), the focus of organizational learning as compared to knowledge management, centers on the capability of a firm to adapt to changing knowledge pressures. Certainly within the field of supply chain management, this argument to develop an organisation's capability towards being an adaptable firm provides the basis for greater flexibility, and hence agility in the long term - factors which can all be inextricably linked to the adoption of information technologies (Swafford, et al. 2008).

It is therefore important to recognize that organizational learning and knowledge management are similar in some ways but have different aims. In particular, the latter seeks to provide people, processes and technology to better manage and make use of intellectual assets; whilst the former is the collective organization-wide realization and usage of knowledge management concepts (Argyris and Schön, 1978; Seely-Brown and Duguid, 1996; Senge, 1990). Thus, it is within this context that the authors seek to investigate and analyze those aspects of knowledge and organizational learning that drive the evaluation and implementation of an ERP system within the given case company. Via the resulting application of a Fuzzy Cognitive Mapping (FCM) approach, further insights into the inter-relationships that exist within a knowledge intensive decision-making task, is achieved.

\section{Research Methodology}

The research question posed is to seek to understand the relationships that exist between KM and $O L$, through analyzing the information systems evaluation decision-making activity. This section describes the methodological stance taken by the authors in approaching this goal, and also details the FCM technique applied.

\section{Research Design}

In deciding upon where to start, the authors adopted the approach advocated by Galliers (1995), where the definition of a morphology or 'form of doing' defines the manner in which the research should be undertaken. As such, and defined by Yin (1994), the authors had to consider the form of the research question being posed in terms of the extent to which the authors would have control over behavioral events; and the degree to which there would be a focus on contemporary events.

It was accepted that the organizational context for the research warranted a multidisciplinary approach. Furthermore, it was acknowledged that to address and craft a model that captured the interplay between $\mathrm{KM}$ and $\mathrm{OL}$ would require a research strategy with inductive characteristics 
(based upon empirical observation in an exploratory and descriptive sense). As such, the deep social nature of the given field of observation meant that there was a need to capture 'rich' primary data in the form of human behavioral and psychological traits and characteristics. However, this paper is not seeking to offer detailed conversations with the interviewees following an interpretivist analysis, nor is it a requirement when developing a rich-picture cognitive map to present such qualitative conversations.

The systematic approach that the authors used to observe the human and organizational ISE process within the selected case company, were based upon a protocol that involved data collection from primary and secondary sources. In doing so, conforming to accepted procedures as defined in the normative fieldwork literature within information systems and social sciences, involving personto-person interviews and analysis of company documentation and archival materials (Fiedler, 1978; Mayring, 2000; Shaughnessy and Zechmeister, 1994; Weick, 1984; Yin, 1956). One-on-one interviews of approximately 1 hour were conducted with the Managing Director (MD), Production Director (PD) and Chief Financial Officer (CFO), as well as numerous shop floor operatives. These people were selected because of their roles within the company but also given their significant experiences. The interviews with the shop floor workers added further credibility to the data generated as well as confirming issues associated with the environmental setting. Notwithstanding, interviewer bias was addressed by cross checking data between interviewees; at all levels.

The use of reflexive feedback as well as the elimination of leading questions through the use of 'probes' (follow-up questions used to get respondents to elaborate on ambiguous or incomplete answers) supported the development of a robust methodology. After every interview, notes were given to each interviewee to check, to resolve any discrepancies that may have arisen and eliminate any interviewer bias. The authors have extensive industrial experience in carrying out research of this type, and have successfully used these methods, as reported by Irani et al. (2005, 1997). To investigate and describe the core issues associated with the evaluation of an information system within a manufacturing setting, the research process is shown in Figure 1.

\section{Insert Figure 1}

Using this diagram as a roadmap of the research process, the focus of this paper is to understand those knowledge factors that have an affect upon the evaluation of an information systems investment within a medium-sized UK manufacturing company. The capture and extrapolation of human and organizational issues form the genesis of a model that is constructed to identify the $\mathrm{KM}$ and $\mathrm{OL}$ interrelationship. In seeking to interrogate these relationships, the authors propose to use a cognitive mapping technique in the guise of the Fuzzy Logic (Zadeh, 1965) technique, of Fuzzy Cognitive Mapping (FCM) (Kosko, 1991). The generation of FCM weight matrices, causal modifiers and an interconnected network of system concepts support the deduction of meaning from results via narrative description. This then allows for the identification of lessons to be learnt from the case analysis presented. 


\section{Modeling Causality: Fuzzy Cognitive Mapping (FCM)}

The technique of FCM is a natural extension to orthodox cognitive maps, which are typically used within the fields of economics, sociology and political science (Axelrod, 1976), and to a limited extent, within Information Systems (Montazemi and Conrath, 1986). An FCM is a method to graphically represent state variables within a dynamical system through links that signify cause and effect relationships; being augmented with fuzzy or multivalent weights that are quantified via numbers, or words (Kosko, 1990, 1991). Visually, an FCM is a non-hierarchic flow graph from which changes to each statement (fuzzy concept, i.e. node), are governed by a series of causal increases or decreases in fuzzy weight values (i.e. links between nodes). The advantage of modeling dynamic systems where there is a great deal of uncertainty, for example, investment decisions, using an FCM, is that even if the initial mapping of the problem concepts is incomplete or incorrect, further additions to the map can be included, and the effects of new parameters can be seen (thus, providing a holistic picture of the scenario being modeled). The causal interrelationship mappings that are linked, provide the basis for analysis via computational means, and can be used as an $\mathrm{Al}$ system which learns from these cognitive inputs. Given an FCM embodies a number of concepts, $C_{i}$ (where $i=1, \ldots, n$ ), this represents a decision-making scenario, consisting of a number of nodes. Any change to the value of each node, is computed from the values of the nodes in the preceding state, using the following equation:

$$
C_{i}^{t+1}=f\left(\sum_{j=1}^{n} W_{i j} C_{i}^{t}\right)+C_{i}^{t-1}
$$

where $C_{i}^{t+1}$ is the value of the node at the $t+1$ iteration, $C_{i}^{t-1}$ is the value of the node at the $t$ 1 iteration, $f$ is a given threshold or transformation function, $W_{i j}$ is a corresponding fuzzy weight between two given nodes, $i$ and $j$, and $C_{i}^{t}$ the value of the interconnected fuzzy node at step $t$. The threshold function, $f(x)$, can be constructed as being bivalent $(x=0$ or 1$)$; trivalent $(x=-1,0$ or 1$)$; hyperbolic (usually tanh $(x)$ ); or the sigmoidal / step function $\left(x=1 / 1+e^{-c x}\right.$, where $c$ is a constant). This threshold function allows the corresponding output of equation 1 to be scaled for ease of visualisation. Tsadiras (2008) has recently reported that each type of function has different dynamic capabilities such that the use of FCMs based upon bivalent and trivalent threshold functions, are more amenable to modeling qualitative scenarios where representation of increase, decrease or stability of a concept is required; but noting that hyperbolic-based FCMs allow a better representation of qualitative and quantitative modeling components and where there is a degree of strategic planning and analysis to be carried out. This has also recently been confirmed by Bueno and Salmeron (2009). In this instance, the authors therefore maintained the usage of the hyperbolic, i.e. $\tanh (\mathrm{x})$ function. The dynamic simulation of an FCM requires the additional definition of the fuzzy weights, $W_{i j}$, within a connection matrix, $W$, and the initial or starting input vector at time $t$, 
$C^{t}$. These weights signify the causal relationship between each concept node. As such, the latter is a $1 \times \mathrm{n}$ rowvector with the values of all concepts, $C_{1}, C_{2}, \ldots, C_{\mathrm{n}}$ for $n$ concepts or nodes in the FCM, whilst the former is a $n \times n$ matrix of weights between any two fuzzy nodes, $w_{i j}$. If there is no direct relationship between the $i^{\text {th }}$ and $f^{\text {th }}$ nodes, then the value of the connection strength is zero. The simulation proceeds by computing $C_{i}^{t+1}$ based upon the initial starting vector, and the given threshold function in $f$, as well as the causal connection strengths in the $n \times n$ matrix, $W$.

Each subsequent iteration then uses the values of the preceding FCM output, much like as in the case of other artificial intelligence techniques as in the case of Tan et al. (2006) who used a combination of case based reasoning and a neural network to learn incrementally from experiential data. The values within this vector signify the activation level of a node in the FCM (i.e. a set of training or goal data). Hence, each $w_{i, j}^{n}$ value defines an initial static state of the FCM, for which each node is set to an "on", "off" or other intermediary position. Therefore, by calculating each subsequent value of equation (1), the FCM simulates the dynamical system being modeled. Each corresponding causally linked node within the mapping then reacts and responds to its respective inputs - the state of each, in a cumulative sense, presage any underlying modality or hidden 'pattern of inference', which belies the implicit system dynamic of the FCM. Thus, the iterative application of equation (1) describes a machine learning process. The process for generating the FCM simulation results is as shown in the lower half of Figure 1.

As such, the authors aim to use this FCM technique to explore the relationship between KM and OL, by principally: elucidating and codifying those key factors involved in the ISE process within the case company; and through the subsequent mapping and analysis, synthesising the case data against this mapping within the context of outlining pertinent explicit and tacit knowledge factors.

\section{Case Study Description}

Many companies have implemented, or are considering implementing manufacturing systems based upon ERP or Extended ERP (ERPII) systems (Koh et al., 2008), to support the formal planning and control of business processes (Sharif and Irani, 2001). Evaluating and assessing the impact and utility of such technologies, is therefore an important task that must be based upon knowledge of the organization. In doing so, the purpose of any justification process is to identify a relationship between the expected value of an investment and an analysis [often quantitative] of the benefits, costs, and risks. The case organization studied in this research, SME-UK, is a manufacturing organization within the UK, which specializes in the manufacture of bespoke aerospace, automotive, and other engineering components.

What differentiates SME-UK from most other companies in its sector and geographic location is the focus on people, process and technology. The interplay here is how SME-UK was seeking to achieve its goals is evident between the motivations to develop a robust IT/IS infrastructure and, the 
company's focus on people, process and technology. Moreover, there was also a mandated focus on process improvement and IT/IS infrastructure enhancement as the basis for improving competitive advantage and instigating an appropriate environment for organizational learning, being a big believer of the principles of Kaizen (Imai, 1986). At the time of conducting the case enquiry, this investment would enable SME-UK to maintain competitive advantage through the innovative use of an ERP system. Figure 2 shows the given strategy map (Kaplan and Norton, 2004) of the organization which includes the vision, mission, values, strategic goals and core processes.

\section{Insert Figure 2 here}

This figure provides an indication of those components, which the company hoped would give rise to realizable benefits, to be used to improve organizational efficiencies through reflexive learning and feedback between its core processes and its strategic vision. As such, this diagram can be read from either top to bottom or vice versa in order to understand those factors that drove the business. Management explained that by approaching investment in ERP in terms of a strategic innovation programme, required business change activities, across the other core processes of Production, Delivery and Support. By enabling and implementing transformational processes which would enable the strategic goals to be achieved, benefits would then be realizable (and hence would ultimately lead to improve of organizational learning).

SME-UK utilized the CBA approach to better understand the implications of the investment, which attempted to include human as well as costs and benefits factors. This had been used for appraising previous investments (such as for Computer Numerically Controlled, CNC, machinery), and was now to be applied to identify those benefits and costs associated with Production Planning and Control (PPC) and Shop Floor Data Collection (SFDC) modules. However, there was no structure to the analysis of those benefits and costs identified and there was no assignment of financial values to the investment implications identified. SME-UK's prescriptive justification process soon proved itself inappropriate, as it was unable to quantify and qualify the intangible and nonfinancial benefits, and indirect costs (as represented and signified in terms of the firm's vision and mission statement). As a result, an 'act of faith' investment appeared to be the only option available, mainly due to the inexperience of the new management team, who were unaware of how to use non-traditional ISE techniques. SME-UK nonetheless developed bespoke ERP in the vein (in part because they were unable to quantify benefits and costs). The management team was also motivated to adopt this strategy because they saw the massive potential strategic benefits such a system could bring to improving production control, throughput and teamwork. Management very much viewed project justification as a hurdle that had to be overcome, and not as a technique for evaluating the project's worth in any sort of rigorous terms. This had significant implications during the preparation of the ERP project's proposal, with managers spending much time and effort investigating its technical and financial aspects (in a strategic sense), rather than risk and costs. The remaining project team members tried to address implementation and human resource risks, against estimated cost implications. So, whilst there was a desire to invest and implement in technology, there were, in a sense, opposing knowledge-based views of the justification process. 
Specific stakeholder job functions and software types associated with the adoption of computerised PPC were identified by SME-UK. Yet the management team did not consult, or identify those operational stakeholders responsible for the relevant business processes. There was a lack of representation and involvement by employees from the team that was responsible for selecting and implementing the initial vendor solution. There appeared to be a lack of interest and ownership by the operational workforce in computerizing business processes - a point noted by Vergidis et al. (2008) recently who also noted the challenges involved in identifying tangible benefits of business process modeling and design. The workforce thought that management had a 'hidden agenda' in implementing SFDC, to gather set-up and run times, therefore implying gathering of performance management data. Therefore, issues of Commitment associated with implementing PPC were addressed from a purely technical perspective, later realising the consequences of neglecting the 'softer' side of implementation.

The organizational culture of the firm was very much driven and was mired in a "them and us" environment, with a mild skeptical stance taken by most of the workers there, revolving around a union-based view of the worker-management relationship. The "buying-out" of two senior directors, and their removal from SME-UK's board, presented the organization with a management experience void; although this did evolve the managerial culture within the firm, from a dominating reactive senior management structure, which had a clear hierarchy and was dependent on traditional approaches to manufacturing - to one of a much clearer responsibility-led leadership style. As a result, the firm's mission statement had changed to encompass a partnership between people, technology, customers and suppliers. Also, SME-UK had done little training and education before their implementation of the PPC/SFDC. When SME-UK purchased their PPC software, the company got 5 day's worth of vendor training, predominantly confined to office end user stakeholders. However, the later implementation of SME-UK's SFDC system, was done in isolation to vendor support, when the system became 'operational', there was much resistance to its use due to a general lack of knowledge about the system, notably commented that people were not informed of the impact of the system and how it would impact their job function. As such, very few people endorsed or understood what the success of this system could bring to the organization.

Operational stakeholders were unaware of the link between SFDC and PPC, which resulted in an unreliable Master Production Schedule (MPS). SME-UK then instigated a series of intensive education sessions where all managers were educated on the impact that the investment would make to their job function(s) and shop floor stakeholders were taught how to use the ERP system within cross-functional teams. Initially, there was considerable management commitment in SMEUK's implementation of vendor PPC/SFDC software. The project was championed by the MD, and when asked why other more directly affected managers were not responsible for leading the project, the MD replied that as he was the main visionary leader, it was up to him to set the standard, and lead the way. However, the MD quickly turned his attention, appearing to have either lost interest, due to implementation problems, or a lack of success, or being 'driven' by other organizational improvement initiatives. Responsibility of the remaining implementation was then delegated to the well-established Production Director. Interestingly, the Production Director was not a key member of the vendor PPC/SFDC implementation team but operated as an honoree, advising on technical 
issues when consulted. Although the Production Director acknowledged the contribution the PPC/SFDC system was making/could make towards the streamlining of the production function, he noted that is was never his project and that he did not relish the possibility of being responsible for mounting cost over-runs.

When SME-UK decided to abandon the project and began developing bespoke ERP, many of the problems associated with management commitment were addressed. In doing so, the new project of bespoke system development was this time supported by the MD, with the Production Director now leading the project. The company by and large recognized that change had to occur across all levels of the organization in terms of people, process and technology. However, this factor was largely at the expense of losing focus and showing a lack of commitment on the details of stakeholder involvement, processes for ISE and communication and system rollout. Furthermore, this given analysis compares favorably with available industrial management experience also, which highlights seven key ERP "problem areas": inadequate executive strategy; weak governance; lack of attention to business process management; lack of commitment to new support for process and system; organizational flaws or inadequacies; IT configuration issues; and infrastructure shortcoming.

\section{Analysing Case Study Experiences via an FCM}

The overall approach that the researchers wished to use in this study was to codify the experiences and knowledge of the case data participants. A graphical representation of resulting facets, a cognitive map was then created which essentially defined the decision-making environment itself. The authors' intention was to observe and analyse the dynamic behavior of this real-world mapping, by applying an iterative algorithm to advance the state of this system. Hence, the application of the FCM technique is now presented, to highlight its use as tool for providing insights into the relationship between $\mathrm{KM}$ and $\mathrm{OL}$ concepts, based on a case description.

\section{Implementation of the research design}

As noted in the research methodology section, the generation of an FCM requires that the given decision-making or knowledge scenario to be modeled, be broken down into some constituent components or nodes. In terms of this study, data was gathered from the senior management and project implementation team. The nodes of the FCM were formulated as a result of feedback and verbalisation process facilitated by the researchers as shown in Figure 1. In considering such factors, the authors felt that it was possible to encapsulate the ISE approach taken in SME-UK, by evaluating fuzzy connectivity (as used successfully in the past also - see Irani et al. (2002). In terms of this research, the objective and intent was to not merely map the ISE decision-making process as a series of causal relationships but then also place this within the context of the particular explicit and tacit knowledge components of the given process investigated. In the latter cases, this was achieved through active participation between both the researchers and case participants within a workshop setting. The authors originally intended to use feedback from employees directly. However, it was found that in the FCM generated their responses were heavily skewed towards a negative causality. As the Production Director shared largely the same sentiments as the 
employees but was aware of other management and organizational factors within with respect to the overall IS evaluation and ERP implementation, it was felt that this represented a more balanced view of the organizational situation. Following this, management were also asked to agree on words that could describe their perception or expectation of a particular part of ISE task (for example, "contributes to", "highly valued"). Once this data was collected by the authors, it was then coded and categorized into key response types: Employee Commitment, Cultural Issues, Training and Education, Management Commitment, Concept / IS Justification, Project Management, ERP Selection, ERP implementation and Vendor Support.

The authors then arranged the causal weights, in terms of these factors into influence matrices. These nodes essentially define the context and scope of the ISE scenario the case company was involved in. The fuzzy connection matrices, are given in equations (2) and (3) for each case (MD and PD respectively). As shown in Table 1 and 2 within these connection matrices, each column and row relates to each of these factors in the order of the response types defined above (from left to right), using the causal modifiers given in Table 3 . Hence, the finalised nodal values within both $W$ matrices essentially define the context and scope of the ISE scenario the case company was involved in and served as the basis for the resulting simulations.

\section{Insert Table 1 here}

\section{Insert Table 2 here}

\section{Insert Table 3 here}

This is shown as the Define and Generate step also in Figure 1. This initial FCM was then shown to the ISE experts within the case company, whereupon feedback from them, the influence matrix was revised as well as a set of ISE scenarios to be used for the FCM simulations (i.e. the Recreate step in Figure 1). For the purposes of this research, this cycle was carried out three times, the details of which are now described in detail.

\section{Computational Results}

The emerging FCMs will act as a tool to aid in the discussion and exploration of the inter-locking enterprise-wide issues associated with decision-making. Using equations (1-3), some representative results of each FCM is presented. As noted in the research methodology section, the generation of an FCM requires that the given decision-making or knowledge scenario to be modeled, be broken down into some constituent components or nodes. The threshold function, $f$, for advancing the FCM simulation as given in equation (1), was set to be the hyperbolic function, $f(x)=$ tanh $(\mathrm{x})$, for all FCMs. This was chosen such that the response would complement and be in line with respect to the numeric range of the causal modifiers. The goal or objective ISE task situations were defined to reflect that used by senior management within SME-UK, in relation to the investment decision, in terms of an Organizational focus and a Human focus (i.e. noting the culture and decision-making approach used before and after implementation). These starting vectors were defined and subsequently adjusted with the help of both the MD and PD participants. 
Each value in these vectors relates to teach of the 9 categories which make up the $W$ matrices in equations (2) and (3), and are a representation of what management consider to be the prime drivers behind each organizational and human foci. Thus, the initial FCM data, or starting row vector $C^{0}$, was set accordingly as follows:

Organizational (i.e. IT/IS) focus:
$[-1.0001 .000$
$-0.500 \quad 0.250$
$-0.250$
$-0.250$
0.500
0.250
$0.000]$

Human (i.e. people) focus:

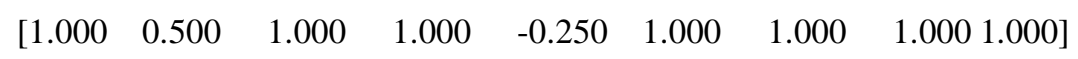

The values within each of these vectors correspond to the nodes given in the order Employee Commitment, Cultural Issues, etc. Taking an organizational focus, highlights and brings into play only those systematic or process-driven issues which are thought to be of primary managerial concern. Likewise, human focus involves the consideration of and weighting towards those factors which are predominantly governed by strong individual or social interactions (such as training, culture and the like). The values within each of these vectors correspond to the nodes given in the order at the start of this section. These initial starting vectors were again, initially defined by the authors and subsequently adjusted with the help of both the MD and PD participants. Each value in these vectors relates to teach of the 9 categories which make up the $\mathrm{W}$ matrices in equations (2) and (3), and are a representation of what management consider to be the prime drivers behind each organizational and human foci. Based upon these details, the fuzzy cognitive maps are shown in Figure 3 and 4.

\section{Insert Figure 3}

\section{Insert Figure 4}

Within these causal maps the given nodes relate to the elucidated case data factors: EC (Employee Commitment), Cl (Cultural Issues), MC (Management Commitment), CJ (Concept Justification), TE (Training and Education), PM (Project Management), ES (ERP Selection), EI (ERP Implementation) and VS (Vendor Support). As can be seen by comparing each FCM against the other, the MD FCM in Figure 3 tends to confirm and uphold the views of management in the sense of wishing to drive the growth of the firm through a commitment to training and education. This is shown in terms of the many causal links from MC to TE. Similarly, there is a concentration of causal links to PM, ES and El factors - denoting a systems-focused (organizational) view of the ISE task being undertaken. In contrast the PD FCM in Figure 4, offers a wider set of interconnecting causality links tending to focus on a plethora of issues, not only TE. Here, there appears to be an 
even balance between both organizational-focused factors (for example, ES, EI, VS, PM) and human factors (for example, EC, $\mathrm{Cl}$ and TE).

To generate the simulation results, the authors used a spreadsheet-based model employing matrix multiplication and graph drawing add-ins in Excel (Volpi, 2004). This was done in order to both generate the directed (di)graph representation and in order to run cognitive simulation scenarios based upon causal weightings and input vector states. Finally, the analysis was then used in order to synthesize the context of knowledge management and organizational learning and develop a model to encapsulate these two concepts. The results are shown for each FCM in Figures $5 a, b-6 a, b$, each result being now discussed in detail (Managing Director and Production Director views). The FCM results in Figure $5 \mathrm{a}$ shows the view of the Managing Director in relation to the Organizational focus row vector in equation (4) and the weight matrix of equation (2). As can be seen, it takes 7 iterations for the system to converge to a limit cycle, with strong alternating cyclical behavior present between training and education, vendor support, employee commitment, project management and concept / IS justification.

\section{Insert Figure 5a}

As such each of these component parts of the FCM is out of phase with one another, indicating an inverse relationship between them. This may highlight the fact that each of these constituent factors leads and / or lags the other, thus signifying that an on-going stimulus-response is required from each of the factors. As such, this can be said to be the hidden pattern of inference within this FCM. Looking at the initial iterations, it can be seen that the FCM displays some level of "conversation" between each key facet. Upon closer inspection, this shows that employee commitment is driven and buoyed by management commitment initially.

The emergence of an increasing level of cultural issues (between iterations $5-7$ ), can be also seen to be prominent and could be said to be based upon weak, varying training and education and ERP implementation factors (noted as the largely negatively sloping curves for each of these aspects between iterations $1-6$ ). It is also interesting to note that the poor application of concept and IS justification were detrimental to the ERP implementation. As such in terms of this FCM view, from iteration 7 onwards it can be seen that a combination of project management, vendor support and training and education factors became overriding alternating issues which management were pre-occupied with. These factors are non-convergent, thereby highlighting the intractability of attempting to define a finite state for them. In practical terms, this may signify a continuous process / cycle, which exists between these factors. It is crucial to note that convergence / non-convergence of an FCM to a fixed point or limit cycle (or any other behaviour), is not a de-facto result which is sought. The aim of an FCM is to show the interplay between the representations of the concept nodes, in order to explore the potential patterns of inference within the system being modelled. This was to the detriment of cultural and employee issues which to all intents and purposes, were weakened (i.e. came to rest at the steady state of -1 , hence possessing a weak causal effect based upon the causal weighting scale provided in Table 1). 
In contrast, the response of this MD FCM to the human focus input produces a much more dam/ped, "calmer" response across all factors, with a limit cycle being reached within 4 iterations in this instance - this is shown in Figure 5b).

\section{Insert Figure 5b}

Noting the points raised previously, this FCM shows that by including a bias towards those individual or socio-cultural issues involved in the implementation of the ERP system within the company, a more harmonious inter-relationship between the key driving ISE factors can be discerned. Primarily, it can be seen that the non-zero response of vendor support, ERP selection and implementation likewise generates a positive environment for training and education (i.e. the increase in response of training and education between iterations 2 and 4). This likewise creates a strong response in terms of increasing the causal effects of cultural issues, project management, concept justification and management commitment issues. These factors themselves settle into a recurring limit cycle beyond iteration 7 in the same vein as the FCM induced with the organizational case in Figure 6a. An alternative view of the ISE situation is offered in the FCM of the production director as shown in Figure 6a, which is based upon the weight matrix in equation (3). This clearly shows a large amount of dynamic and chaotic responsiveness for a number of iterations, and it is only after iteration 11 that the system begins to converge to a fixed-point cycle (at iteration 31 ).

\section{Insert Fiqure 6a}

The key aspect of this particular FCM which is driven by the organizational stimulus, i.e. the IT/IS or process-focused approach to evaluating and implementing ERP within the organization, is the perception of the gradually deteriorating causal effect of training and education.

It was this lack of initial focus on training and education needs that lead to the prolonged and ultimately unsuccessful rollout of the SFDC module within the company. However, looking at the response of the FCM it can also be said that the improving, positive causal influence of continued management commitment to fix the initial problems with ERP implementation, did provide an environment and basis for "dampening" the chaotic response of the remaining ISE factors (i.e. the convergence towards a positive, non-zero causal value of 1 for all of the FCM components apart from training and education from iteration 13 onwards). Examining the response of this FCM up till iteration 13 shows a wide variation and interplay between all of the FCM factors. From this response, it appears that management commitment is out of phase with all of the other factors (signifying a potential misunderstanding by management of the key issues that mattered within this decision-making scenario). On the other hand, the overall positive trend and stabilizing influence of the project management approach taken did tend to lead the other factors towards a positive causal state.

Figure $6 \mathrm{~b}$ shows the human-focused view of the ERP project by the production manager also, being based on the initial state vector in equation (5). Here, once again, a much more damped and less chaotic response is shown, whereby a fixed-point cycle is reached within the shorter timescale 
of 27 iterations. The training and education factor still has a negative causal influence overall, and within the first 10 iterations at least, employee commitment leads the responses of ERP implementation and cultural issues and is not too out of sync with the management commitment response. By including such human and organizational factors as part of the ISE overall, provides a faster stabilization and understanding of the key issues involved in this decision-making process.

\section{Insert Figure 6b}

These results show that, essentially, management's view of the decision-making process and implementation of the enterprise system, were at odds to those of middle management as individuals process the information they receive to make a decision in different ways (Barkhi, 2002). This was in terms of how employee commitment and organizational culture was viewed. Ultimately, the managing director was more concerned with ERP selection and project management, as opposed to the human (shop workfloor) issues, which the production director was more concerned about, and was in tune with. The causal flows resulting from such a walkthrough of the modelled situation, if plotted as an FCM, would potentially show these factors at the center of all the other decision-making criteria. This particular set of causal flows investigated, has highlighted the disparity and disconnect that can occur between managerial decision-making and consumers of IT/IS within organizations. Although this has been limited to a single set of conditions and views of the investment appraisal process, this technique has shown that pertinent lessons can be learnt from plotting key operational, tactical and strategic goals within such MIS decision-making.

Thus, at a fundamental level, FCMs provide a mapping of knowledge, and is thus a network visualization of domain expertise and factors that drive the utilization of that knowledge. When comparing both MD and PD FCMs using the Organizational focus rowvector as the FCM stimulus (Figures 5a and 6a), shows that taking this as a basis for ISE shows short-term high activity and almost a chaotic response. Similarly comparing MD and PD FCMs with the Human focus rowvector (Figures $5 \mathrm{~b}$ and $6 \mathrm{~b}$ ), provides a more stable decision-making environment inter alia of other concerns. This is akin to stating that by introducing systematic (i.e. explicit knowledge) issues over those which include and human and organizational (i.e. tacit knowledge) factors, leads to a prolonged, negatively-skewed causal outcome, where employee and cultural factors have little bearing on the ISE outcome. As shown in the FCM results, even if the given non-process view of the ISE situation is taken into account, the lack of planning and execution of training and education continues to be an outlier that needs to be addressed, and learnt by the organization. If the combined FCM results are viewed as being the normalized response in general, it can be seen once again that explicit use of management commitment and project management has a direct interrelationship with employee commitment, training and education, cultural issues ERP implementation and vendor support. Thus, it can be said that those factors that appeared to be detrimental were in fact supporting the project.

\section{Insert Table 4}


This table relates both FCM results with given KM and OL concepts interpreted from the given literature in these fields and the field data from SME-UK. This table was generated as a result of analysing the congruence between the FCM nodes and SME-UK's learning catalysts. This was done in order to provide closure to the goal cited in the beginning of this paper, of attempting to relate $\mathrm{KM}$ with $\mathrm{OL}$ within an organizational setting. By identifying common enablers (the intersecting rows and columns of this table), the authors have been able to identify and segregate the strategic goals of SME-UK, into constituent KM and OL parts (with an overlap between the management of technology and the implementation of strategic goals required). Furthermore, in doing so, the authors have also sought to provide the spectrum of business processes, which underlie KM (in the sense of IT implementation-related knowledge: systems, processes, data); through to organizational culture (employee-management relations, team skills, and other stakeholder issues as identified). This is shown as "Knowledge Basis" and "Business Process" rows at the bottom of the table. The table contents can be read as follows. For example, to adopt technology for competitive advantage (one of the SME-UK's strategic goals); with respect to Management Commitment, Concept Justification, Vendor Support, Project Management, ERP Implementation; requires Technological Change, Technology Management and Strategic Vision to occur. In other words, strategic goals can be matched against decision-making concepts (i.e. the FCM nodes), in order to elucidate particular knowledge forms (in terms of a $\mathrm{KM}$, a mixture of $\mathrm{KM} / \mathrm{OL}$ or purely an $\mathrm{OL}$ basis). It can be seen that the results presented so far, that in the case of SME-UK, there was little or no reward system or incentive given, which as such did not allow organizational learning to take place immediately.

\section{Conclusions}

In interpreting the case data, an interplay of sociological, behavioural and ISE knowledge factors have been highlighted - for which the inherent, tacit relationship and pattern of inference resulting from the application of an FCM has presented management commitment and project management as key driving issues. In light of these observations and analyses, the authors were able to formulate a model that showed the relationship between those knowledge management and organizational learning concepts within SME-UK. The model developed showed that a relationship does exist between $\mathrm{KM}$ and $\mathrm{OL}$ and in this case, each knowledge concept engenders / fosters realization of the other. A consistent involvement and balancing of systematic and behavioural issues can only allow the ideal of organizational learning to be realized. As has been shown however, there may be many tacit as opposed to explicit factors that may achieve this (the FCM being used as a tool to facilitate this discovery). The proposed model not only highlights those factors included in the particular ISE but, also subsequently defines a potential basis for creating a learning organization.

The decision-making approach used by SME-UK was abstracted, with interdependencies then modeled. Simulation results were generated that provided an insight to the interplay between KM and $\mathrm{OL}$, when contextualized through human and organizational factors within the information systems evaluation process. These results presented demonstrate: 
- The MD FCM shows project management, vendor support and training and education as being implicit (tacit) knowledge factors for the ISE process. The former underlying factors likewise provided a causal relationship to the training and education, management and employee commitment concepts.

- The PD FCM shows that project management was an important facet of this case but noted that a combination of consistent management commitment produced a stabilizing effect on the eventual outcome of the ERP implementation. This highlights the importance of management intervention, responsibility, and governance (although at the expense of training and education).

It appears from the FCM results, that issues that were experienced and seemed to inhibit implementation success (management commitment and project management) were in fact inherent, and tacit to the actual outcome of the EPR project. This was however, implicitly recognized by the company, who also realized later that the involvement of people (at management, employee or even vendor level) was crucial to the outcome of investment in technology. As a result, it appears that SME-UK itself recognised in retrospect, that:

- Training and Education are vital to the continuing success of the company, at both employee level as well as managerial level and should be strategically planned in advance of but with respect to, any change programme;

- Tacit issues were not made explicit (employee commitment and cultural issues festered; initial management decision-making, responsibility and commitment was lacking) - the result of which, inhibited project success;

- Organizational culture needs to develop to encompass a diverse and rich communication channel between management and employees (and likewise, feedback and response relating to technology initiatives should not be ignored).

- Management commitment to any company-wide programmes, needs to be consistent, clear and have the appropriate governance and ownership structures in place in order to avoid conflicts of authority and responsibility (and the avoidance of a 'blame culture').

\section{References}

Argyris, C. Schön, D. (1987). Organizational learning: A theory of action perspective, Reading, Mass. Addison Wesley.

Axelrod, R. (1976). Structure of Decision: the cognitive maps of political elites, Princeton. Princeton University Press.

Dervin, B. (1999). 'On studying information seeking methodologically: the implications of connecting meta theory to method', Information Processing and Management, 35, pp. 727-50.

Ballantine, J. and S. Stray (1999). 'Information systems and other capital investments: Evaluation practices compared', Logistics and Information Management, 12, pp. 78-93.

Barkhi, R. (2002). 'Cognitive style may mitigate the impact of communication mode', Information and Management, 39, pp. 677-688. 
Belkin, N. J. (1980). 'Anomalous state of knowledge as a basis for information retrieval', Canadian Journal of Information Science, 5, pp. 133-43.

Bueno, S., and Salmeron, J.L. (2009). Benchmarking main activation functions in fuzzy cognitive maps. Expert Systems with Applications, 36 : 5221 - 5229.

Davenport, T. H. Prusak, L. (1998). Working Knowledge: How organizations manage what they know. Boston, MA: Harvard Business School Press: Boston, MA, USA.

Fiedler, J. (1978). Field Research: A Manual for Logistics and Management of Scientific Studies in Natural Settings. Jossey-Bass, San Francisco, CA.

Galliers, R. D. (1995). 'Choosing information systems research approaches'. In: Galliers, R. D. (eds.) Information Systems Research - Issues, Methods and Practice Guidelines, pp. 144-162, London, UK, Blackwell Scientific.

Hackney R A, Desouza K \& Irani Z (2007) 'Constructing and Sustaining Competitive Inter-Organizational Knowledge Networks: an analysis of managerial Web-based facilitation', Information Systems Management Journal, 25(4): 356-363.

Handy, C. (1990). Understanding Organizations. Penguin, UK.

Imai, M. (1986). KAIZEN: The Key to Japan's Competitive Success. CA: McGraw-Hill.

Irani, Z. and A. M. Sharif (1998). 'A revised perspective on the evaluation of IT/IS investments using an evolutionary approach'. Proceeding of the Late Breaking Papers at Genetic Programming, Madison, WI, University of Wisconsin, July 22-25, p. 77-83.

Irani, Z. and A. M. Sharif and P. E. D. Love (2005). 'Linking Knowledge Transformation to Information Systems Evaluation', European Journal of Information Systems, 14, pp. 213-228.

Irani, Z. and P. E. D. Love (2001). 'The propagation of technology management taxonomies for evaluating information systems', Journal of Management Information Systemsm, 17, pp. 161177.

Irani, Z. and P. E. D. Love (2002). 'Developing a frame of reference for ex-ante IT/IS investment evaluation', European Journal of Information Systems, 11, pp. 74-82.

Irani, Z., A. M. Sharif, P. E. D. Love and C. Kahraman (2002). 'Applying Concepts of Fuzzy Cognitive Mapping to model IT/IS Investment Evaluation', International Journal of Production Economics, 75, pp. 199-211.

Irani, Z., J.M. Sharp and M. Kagioglou (1997). 'Improving business performance through developing a corporate culture', The International Bi-Monthly for Total Quality Management: The TQM Magazine, 9, pp. 206-216.

Irani, Z., S. Jones, P. E. D. Love, T. Elliman and M. Themistocleous (2005). 'Evaluating Information System Investments in Local Government: Drawing Lessons from Two Welsh Cases', Information Systems Journal, 15, pp. 61-82.

Schumpeter, J. (1934). The Theory of Economic Development: An enquiry into Profits, Capital, Credit, Interest and the Business Cycle. Oxford University Press, Oxford, UK.

Järvenpää, E. and S. Immonen (1998). 'Quality of working life in knowledge and information work: Implications for information society'. In: P. Vink, E. A. P. Koningsveld and S. Dhondt (Eds), 
Human Factors of organizational design and management, pp. 195-200. Amsterdam, NL: Elsevier.

Kaplan, Robert S. 1986. 'Must CIM be Justified by Faith Alone?' Harvard Business Review, 64(2): 87-95.

Kaplan, R. S. and D. P. Norton (2004). Strategy Maps: Converting Intangible Assets into Tangible Outcomes. Boston, MA, Harvard Business School Press.

Kess, P., and Haapasalo, H. (2002). Knowledge creation through a project review process in software production. International Journal of Production Economics, 80 : 49 - 55.

King, W. R. (2000). 'Playing an integral role in Knowledge Management', Information Systems Management, 17, pp. 1-3.

King, W. R. and D. G. Ko (2001). 'Evaluating Knowledge Management and the Learning Organization: An Information/knowledge Value Chain Approach', Communications of the AIS, 5.

King, W. R., P. V. Marks and S. McCoy (2002). 'The most important issues in Knowledge Management', Communications of the ACM, 45, pp. 93-97.

Kluge, J., W. Stein and T. Licht (2001). Knowledge Unplugged. Palgrave, Basingstoke, UK.

Koh, S.L., Gunasekaran, A., and Rajkumar, D. (2008). ERP II: The involvement, benefits and impediments of collaborative information sharing. International Journal of Production Economics, 113 (1) : $245-268$.

Kosko, B. (1990). Fuzzy Thinking: The new science of Fuzzy Logic. Flamingo Press / HarperCollins, London, UK.

Kosko, B. (1991). Neural Networks and Fuzzy Systems. Saddle River, NJ: Prentice-Hall.

Leonard-Barton, D. (1995). Wellsprings of Knowledge: Building and Sustaining the sources of Innovation. Harvard Business School Press, Boston, MA, USA.

Levine, L. (2001). 'Integrating knowledge and process in a learning organization', Information Systems Management, 18 (Winter (1)), pp. 21-33.

Lohse, G. L., D. Min amd J.R. Olson (1995). ,Cognitive evaluation of system representation diagrams', Information and Management, 29, pp. 79-94.

Markus, M. L. (2004). 'Technochange management: Using IT to drive organizational change', Journal of Information Technology, 19, pp. 3-19.

Mayring, P. (2000). 'Qualitative content analysis', Forum: Qualitative Social Research, 1, pp. 1-80.

McCampbell, A. S., L. M. Clare and S. H. Gitters (1999). 'Knowledge Management: the new challenge for the 21st Century', Journal of Knowledge Management, 3, pp. 172-179.

Minsky, M. (1975). 'A framework for representing knowledge'. In: P. H. Winston (eds), The Psychology of Computer Vision. New York, NY: McGraw-Hill.

Montazemi, A. and D. Conrath (1986). 'The use of cognitive mapping for information requirement analysis', Manufacturing Information Systems Quarterly, March, pp. 45-56.

Newman, B. and K. W. Conrad (1999). 'A Framework for characterizing Knowledge Management, methods, practices and technologies'. Proc. Documation '99, Toronto, Canada.

Nonaka, I. and H. Takeuchi (1995). The Knowledge-creating Company: How Japanese Companies Create the Dynamics of Innovation. Oxford University Press, UK. 
Nonaka, I. 2004. A Dynamic Theory of Organizational Knowledge Creation, Organization Science, 5(1): 14-37.

Drucker, P. (1993). Post-Capitalist Society, Harper Collins, New York.

Polanyi, M. (1967). The Tacit Dimension. New York, Doubleday-Anchor.

Quillian, M. R. (1967). 'Word concepts: A theory and simulation of some basic semantic capabilities', Behavioural Science, 12, pp. 410-430.

Quinn, J. B. (1992). Intelligent Enterprise: A knowledge and service-based paradigm for Industry. New York Free Press: NY, New York, USA.

Ruggles, R. (1997). 'The State of the Notion: Knowledge Management in Practice', California Management Review, 40, pp. 80-89.

Saracevic, T. (1996). 'Modelling interaction in Information Retrieval: A Review and proposal'. Proceedings of the 59th ASIS Annual Meeting, Volume 33, Baltimore, Maryland, USA.

Saunders, C. and S. Miranda (1998). 'Information acquisition in group decision-making', Information and Management, 34, pp. 55-74.

Seely-Brown, J. and P. Duguid (1996). 'Organizational Learning and Communities-of-Practice: Toward a Unified View of Working, Learning, and Innovation'. In: Cohen, M. D., L.S. Sproull (eds.), Organizational Learning, Sage Publications, Thousand Oaks, pp. 58-82.

Senge, P. M. (1990). The Fifth Discipline, The art and practice of the learning organization, Random House, London, UK.

Shaughnessy, J.J. and E. B. Zechmeister (1994). Research methods in Psychology. 3rd edition, New York, McGraw Hill.

Skerlevaj, M., Stenberger, M.I., Skrinjar, R., and Dimovski, V. (2007). Organizational learning culture - the missing link between business process change and organizational performance. International Journal of Production Economics, 106 : 346 - 367.

Sorensen, C. and M. Kakihara (2002). ;Knowledge Discourses and Interaction Technology', Department of Information Systems Working paper 115, London School of Economics and Political Science, London, UK.

Sunassee, N. N. and V. Haumant (2004). 'Organizational learning versus the learning organization'. Proceedings of the 2004 annual research conference of the South African institute of computer scientists and information technologists on IT research in developing countries, (SAICSIT'04), Stellenbosch, Western Cape, South Africa, South African Institute for Computer Scientists and Information Technologists, Republic of South Africa, pp. 264-268.

Swafford, P.M., Ghosh, S., and Murthy, N. (2008). Achieving Supply Chain Agility through IT Integration and Flexibillity. International Journal of Production Economics, 116 : $288-297$.

Swan, J. (1997). 'Using Cognitive Mapping in Management Research: Decisions about Technical Innovation', British Journal of Management, 8, pp. 183 - 198.

Tan, K.H., Lim, C.P., Platts, K., and Koay, H.S. (2006). An intelligent decision support system for manufacturing technology investments. International Journal of Production Economics, 104 : $179-190$.

Tsadiras, A.K. (2008). Comparing the inference capabilities of binary, trivalent and sigmoid fuzzy cognitive maps. Information Sciences, $178: 3880$ - 3994. 
Vergidis, K., Turner, C.J., and Tiwari, A. (2008). Business Process Perspectives : Theoretical perspectives versus real world practice. International Journal of Production Economics, 114 : $91-104$.

Volpi, L. (2004). 'Matrix 1.8 - Matrix and Linear Algebra functions for EXCEL', Available on-line. http://digilander.libero.it/foxes/index.htm (accessed on 29 August 2007).

Weick, K. E. (1984). 'Theoretical assumptions and research methodology selection'. In McFarlan F. W. (eds), The information systems research challenge, Harvard Business School Press, Boston, MA, pp. 111-132.

Yin, R. K. (1994). Case study research: Design and Methods - 2nd Ed.. Sage Publications, Thousand Oaks,CA: Sage Publications.

Zadeh, L. A. (1965). 'Fuzzy sets', Information and Control, 8, pp. 338-353. 


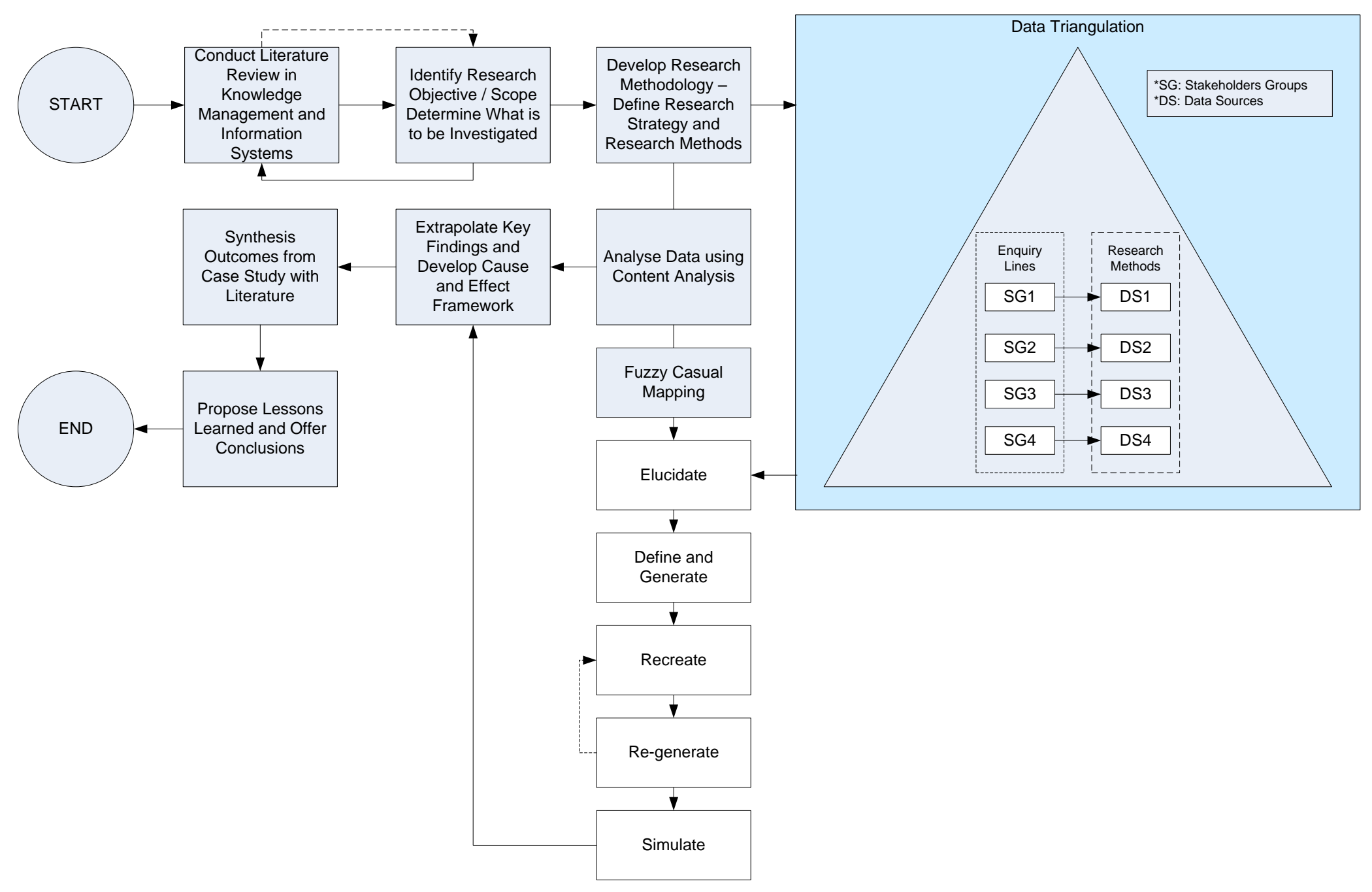

Figure 1. Research Methodology 


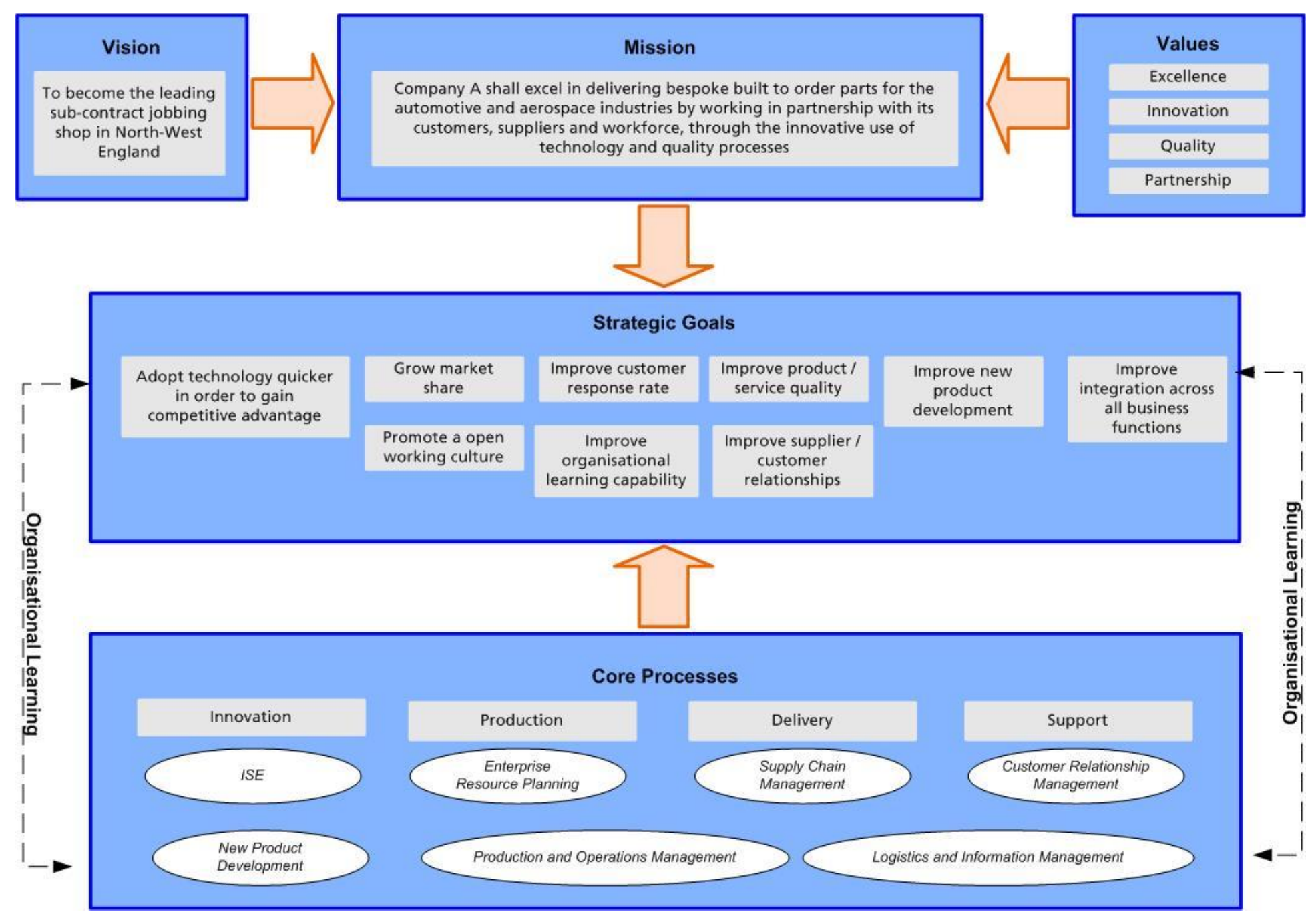

Figure 2. Strategy Map of SME-UK 


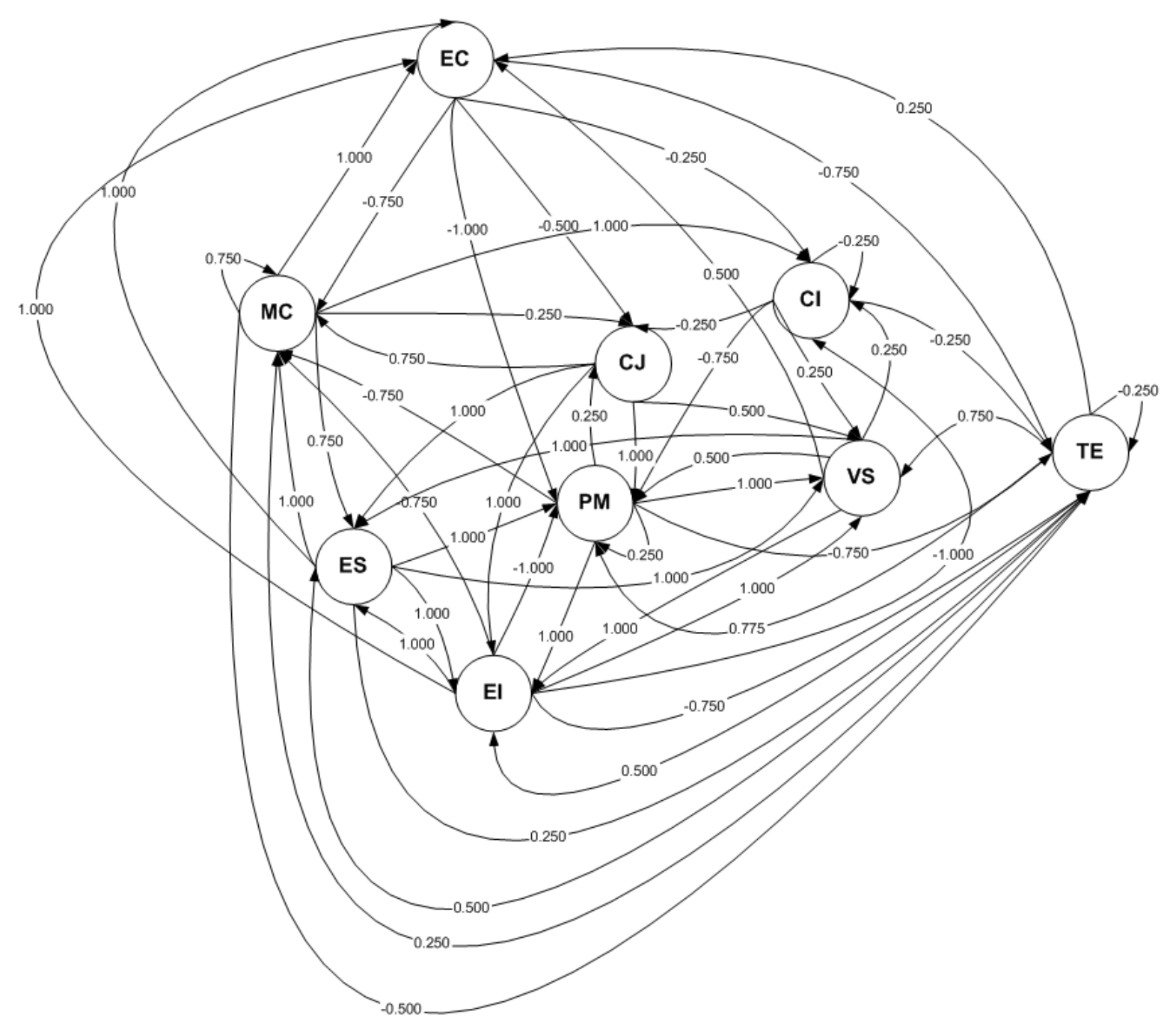

Figure 3. MD FCM 


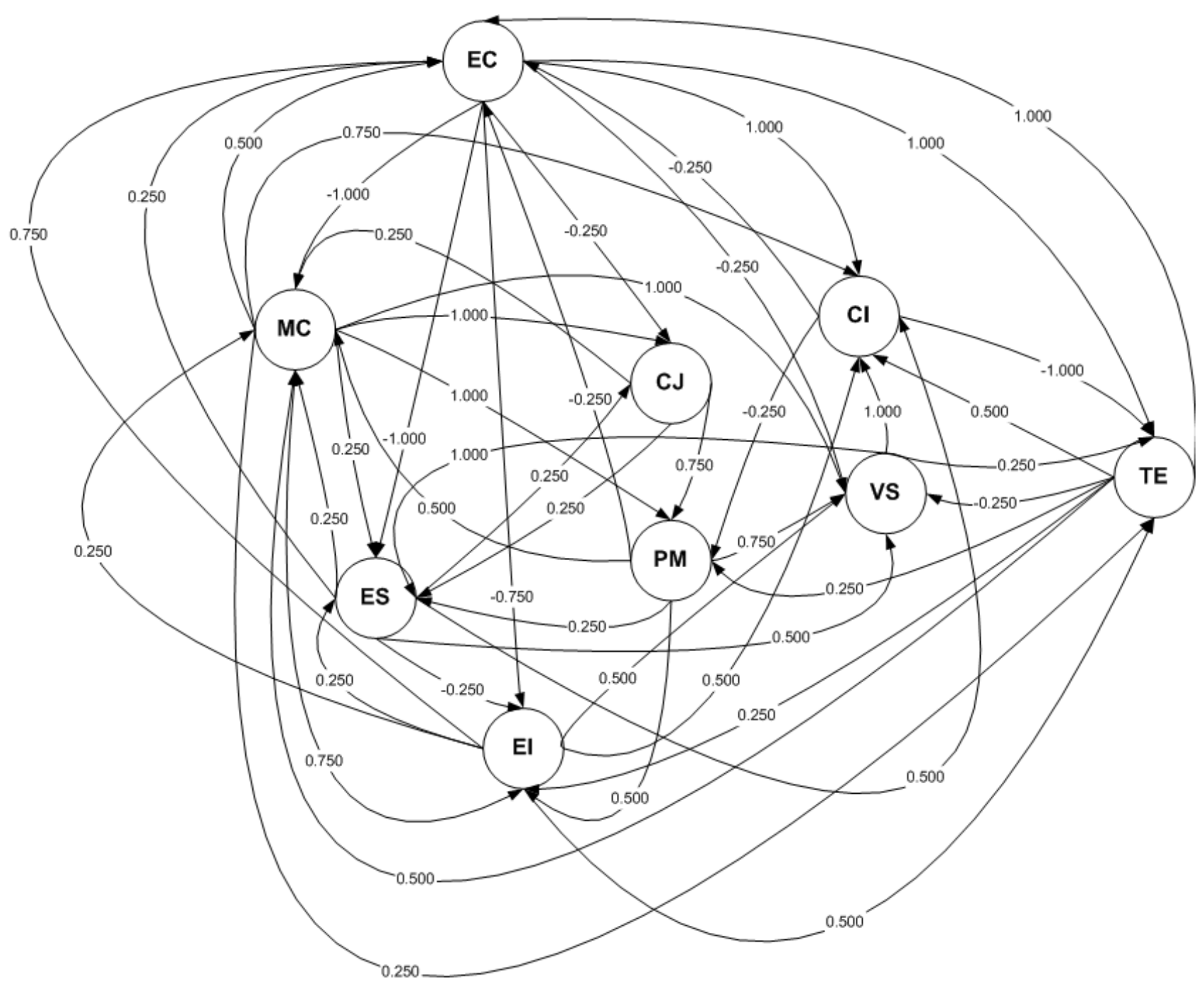

Figure 4. PD FCM 


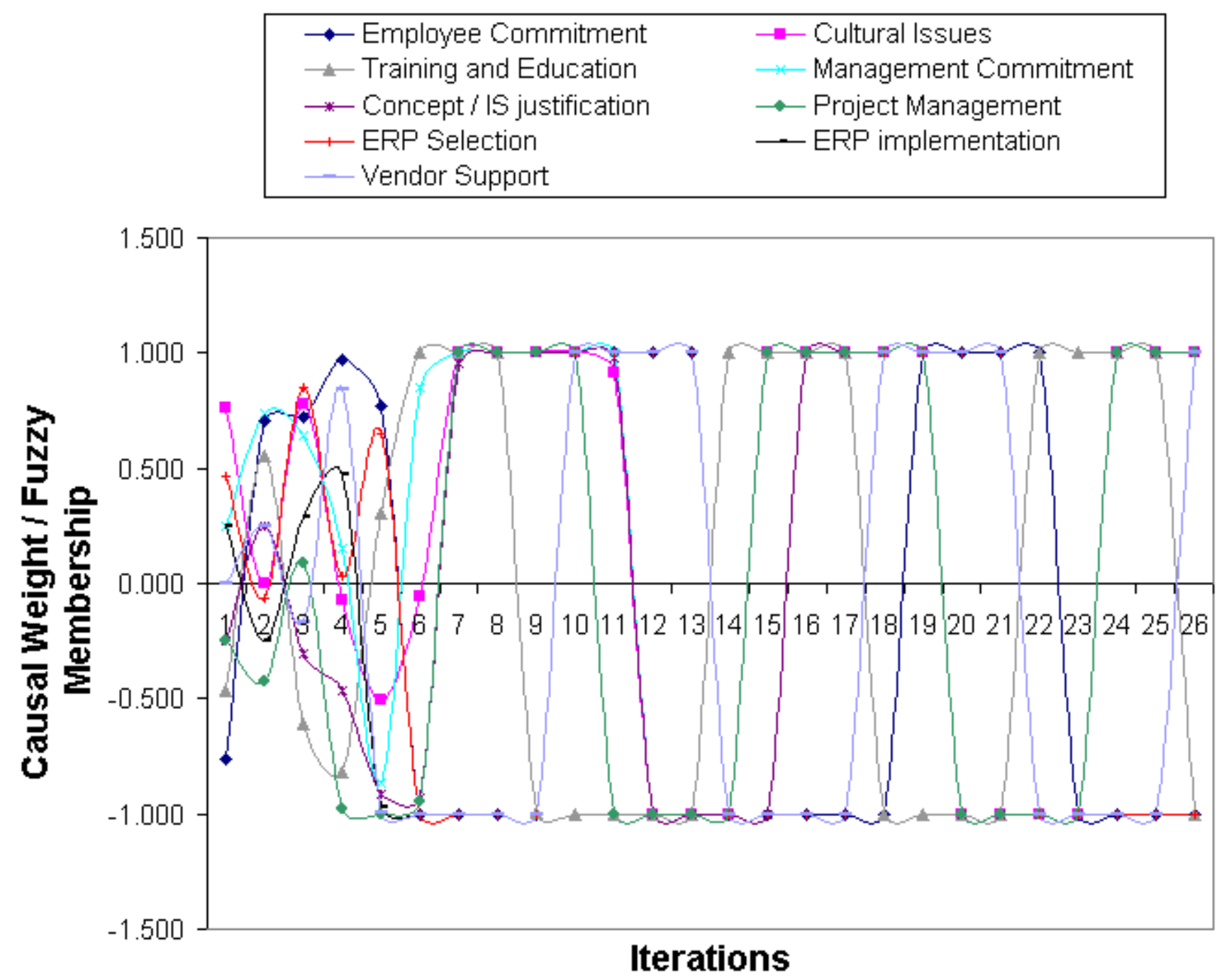

Figure 5a. MD FCM response using Organization Focus 


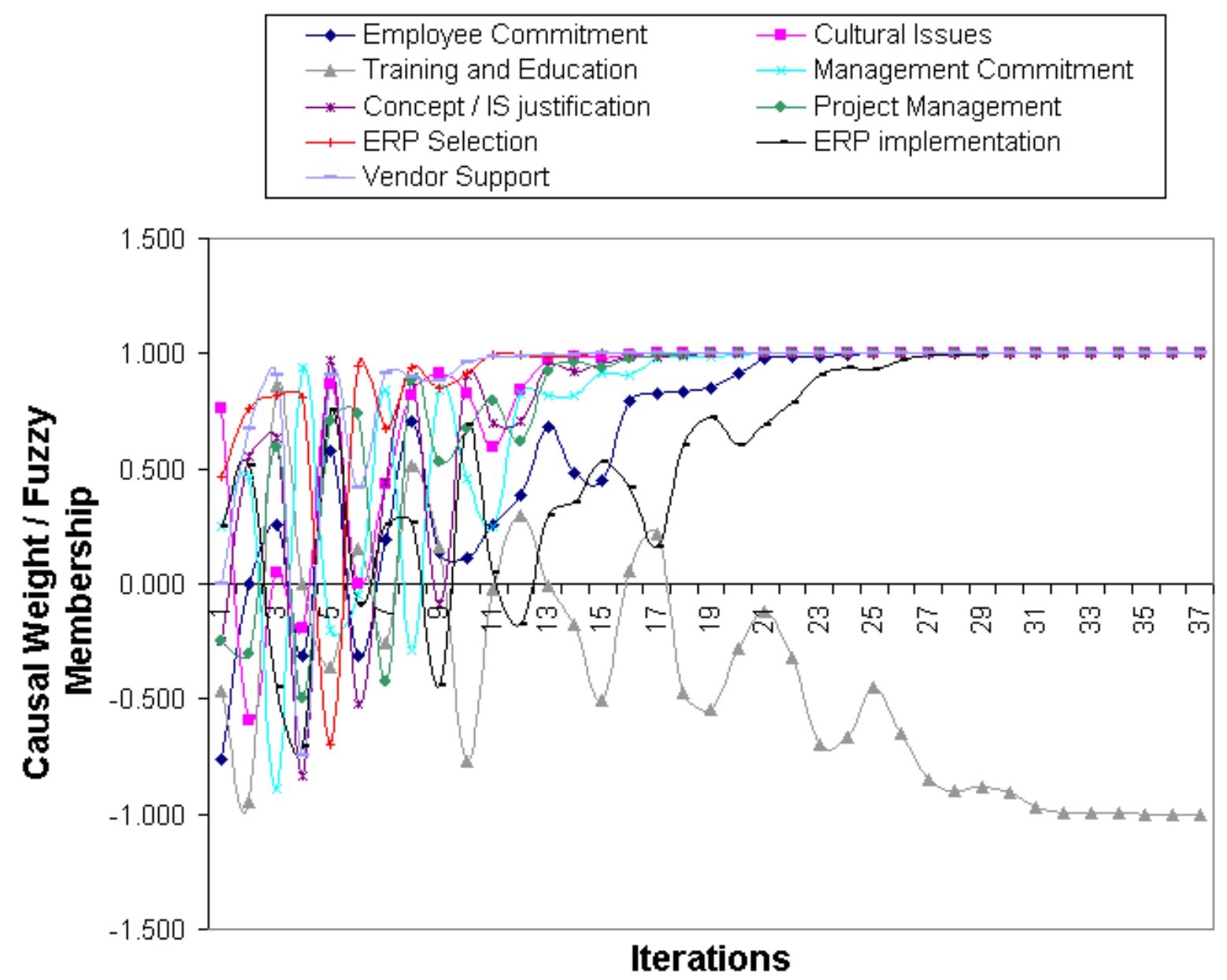

Figure 5b. MD FCM response using Human Focus 


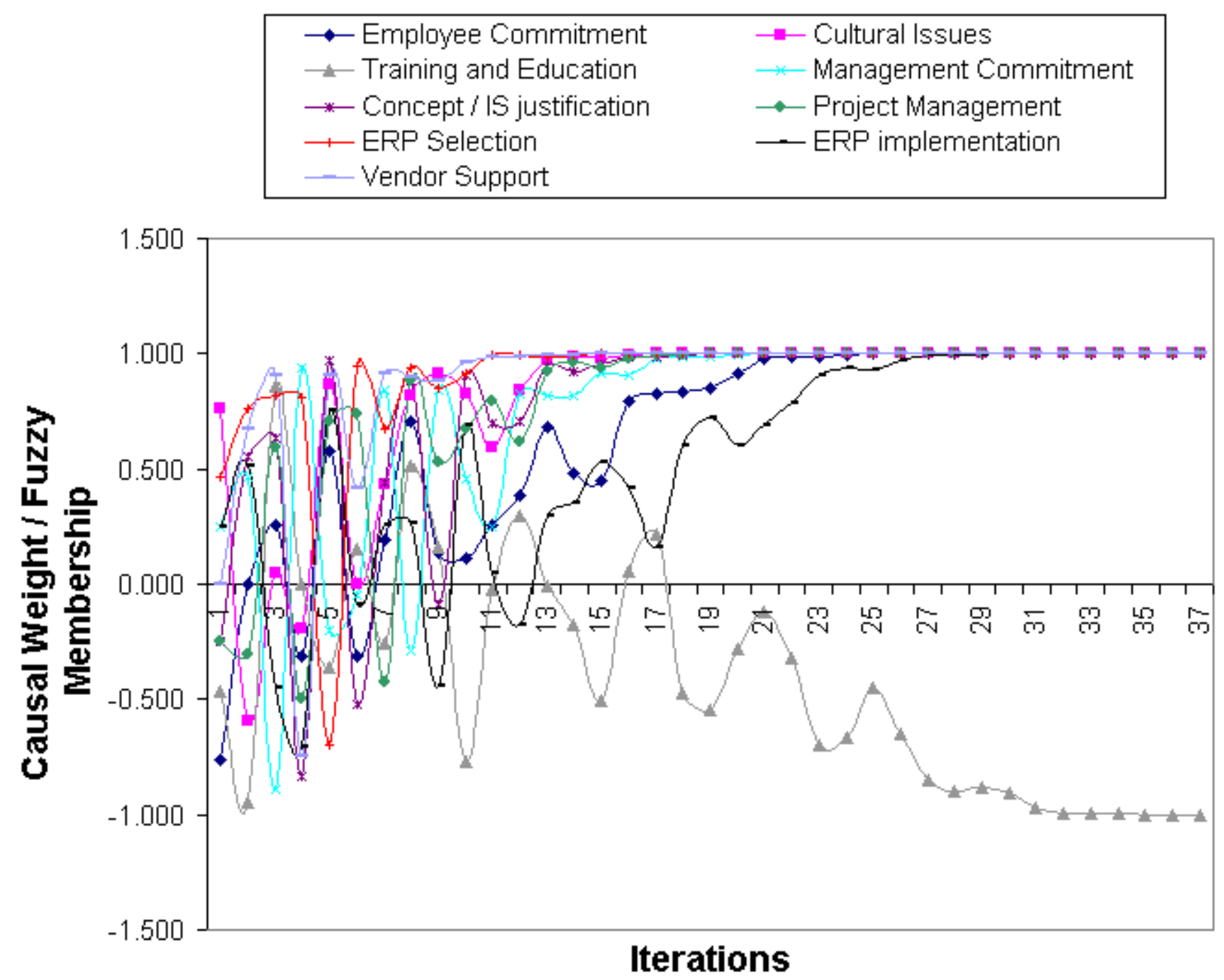

Figure 6a. PD FCM response using Organization Focus 


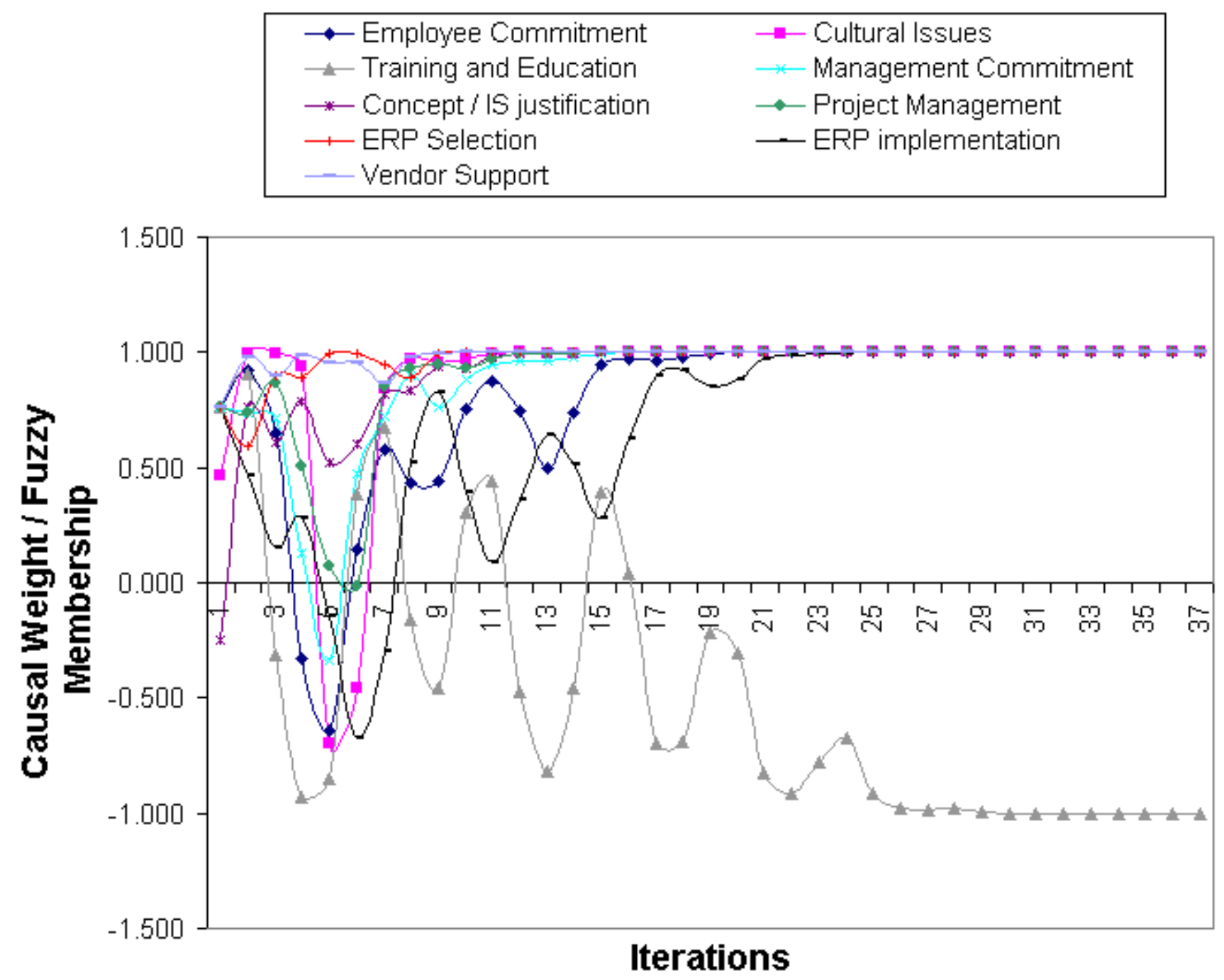

Figure 6b. PD FCM response using Human Focus 
Table 1. Fuzzy connection matrix for MD

\begin{tabular}{|c|c|c|c|c|c|c|c|c|c|}
\hline & 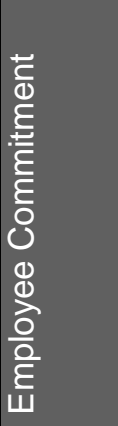 & 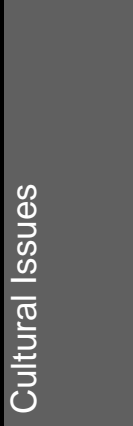 & 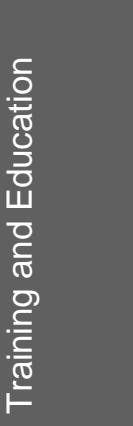 & 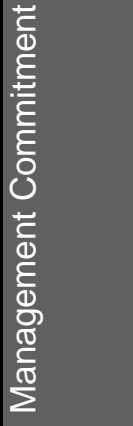 & 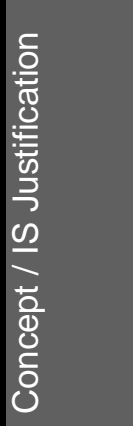 & 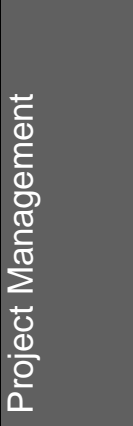 & 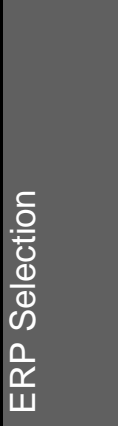 & 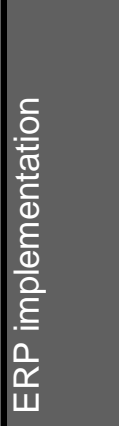 & 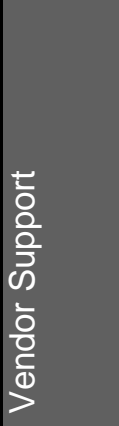 \\
\hline Employee Commitment & 0.000 & -0.250 & -0.750 & -0.750 & -0.500 & -1.000 & 0.000 & 0.000 & 0.000 \\
\hline Cultural Issues & 0.000 & -0.250 & -0.250 & 0.000 & -0.250 & -0.750 & 0.000 & 0.000 & 0.250 \\
\hline Training and Education & 0.250 & 0.000 & -0.250 & 0.250 & 0.000 & 0.775 & 0.500 & 0.500 & 0.750 \\
\hline Management Commitment & 1.000 & 1.000 & -0.500 & 0.750 & 0.250 & 0.000 & 0.750 & 0.000 & 0.000 \\
\hline Concept / IS Justification & 0.000 & 0.000 & 0.000 & 0.750 & 0.000 & 1.000 & 1.000 & 1.000 & 0.500 \\
\hline Project Management & 0.000 & 0.000 & -0.750 & -0.500 & 0.250 & 0.250 & 0.000 & 1.000 & 1.000 \\
\hline ERP Selection & 1.000 & 0.000 & 0.250 & 0.750 & 0.000 & 0.500 & 0.000 & 1.000 & 1.000 \\
\hline ERP implementation & 1.000 & -1.000 & -0.750 & -0.750 & 0.000 & -1.000 & 1.000 & 0.000 & 1.000 \\
\hline Vendor Support & 0.500 & 0.250 & 0.250 & 0.000 & 0.000 & 0.500 & 1.000 & 1.000 & 0.000 \\
\hline
\end{tabular}

Table 2. Fuzzy connection matrix for PD

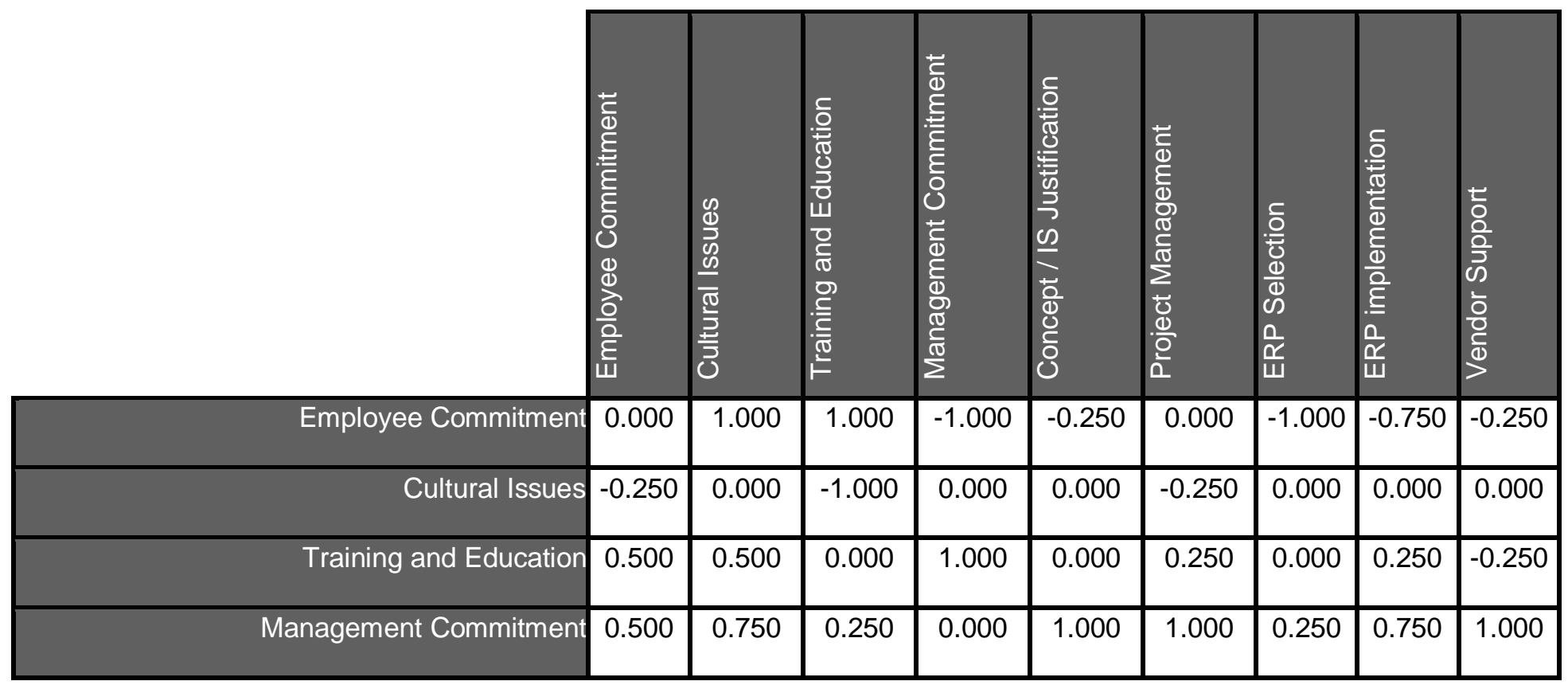




\begin{tabular}{|r|c|c|c|c|c|c|c|c|c|}
\hline Concept / IS Justification & 0.000 & 0.000 & 0.000 & 0.250 & 0.000 & 0.750 & 0.250 & 0.000 & 0.000 \\
\hline Project Management & -0.250 & 0.000 & 0.000 & 0.500 & 0.000 & 0.000 & 0.250 & 0.500 & 0.750 \\
\hline ERP Selection & 0.250 & 0.500 & 0.000 & 0.250 & 0.250 & 0.000 & 0.000 & -0.250 & 0.500 \\
\hline ERP implementation & 0.750 & 0.500 & 0.500 & 0.250 & 0.000 & 0.000 & 0.250 & 0.000 & 0.500 \\
\hline Vendor Support & 0.000 & -0.250 & 0.250 & 0.000 & 0.000 & 0.000 & 1.000 & 0.000 & 0.000 \\
\hline
\end{tabular}


Table 3. Causal weightings

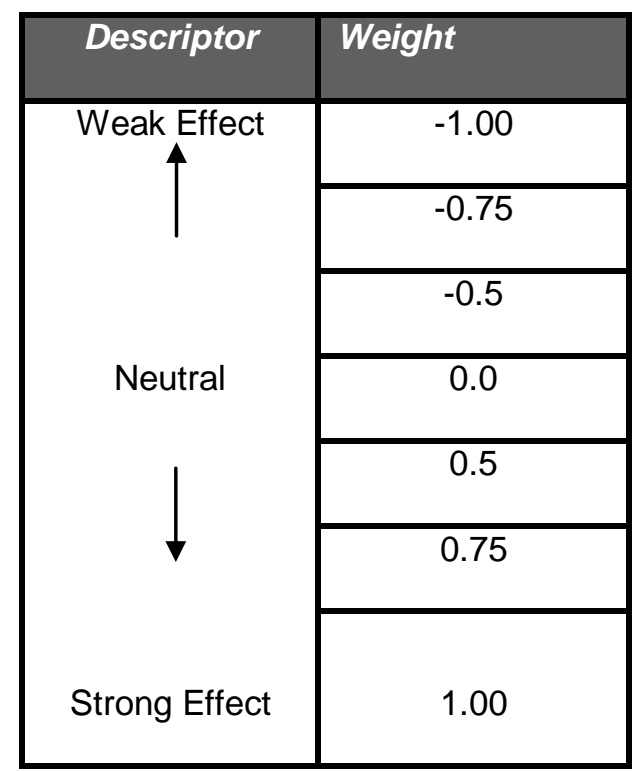


Table 4. Alignment of SME-UK strategic goals against FCM responses

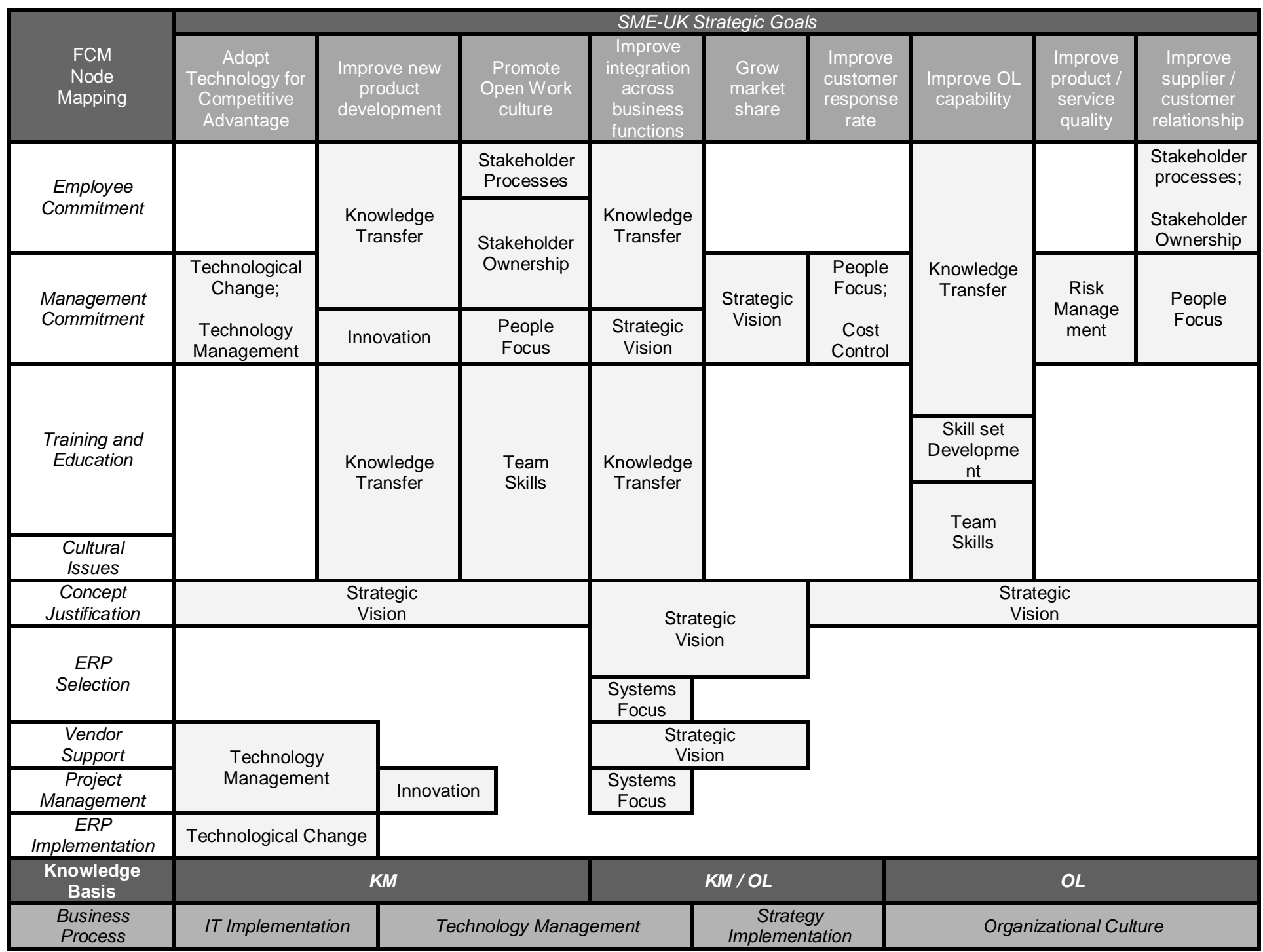

